\title{
MEMS Tunable Asymmetric Fabry-Perot Cavity for High-Precision Weighing of Macro Samples
}

\author{
Alexandre Poulin, Raphael St-Gelais, Ali L. Eichenberger, Luc Thévenaz, Senior Member, IEEE, and \\ Yves-Alain Peter, Senior Member, IEEE
}

\begin{abstract}
We propose a microelectromechanical systems-based tunable asymmetric Fabry-Perot cavity for the high-precision weighing of macro samples. The device is based on an in-plane design and is structured in a silicon-on-insulator substrate. The cavity length of the optical resonator is tuned under the action of an external force. The force can be determined from the resulting spectral shift of the optical resonance. Measurements can be done under static conditions and are immune to electromagnetic interferences. Various designs have been simulated, fabricated and characterized. We report the experimental performances of four devices that have been tested under loads up to $98 \mathrm{mN}$ $(10 \mathrm{~g})$. Sensitivities ranging from 0.51 to $67.69 \mathrm{~nm} / \mathrm{mN}$ and absolute resolution ranging from 0.15 to $19.61 \mu \mathrm{N}$ are reported. The maximum relative resolution of the sensor is below 100 ppm.

[2012-0258]
\end{abstract}

Index Terms-Bragg grating, Fabry-Perot, microelectromechanical systems MEMS), optical microbalance.

\section{INTRODUCTION}

$\mathbf{M}$ OST OF THE microelectromechanical systems (MEMS) can be classified either as actuators or sensors. Performance maps have been drawn to get a global view of these fields [1]-[3]. They allow selecting the best suited type of sensor for a given application and can be used as preliminary design tools in the development of new devices. Force sensors can be used to monitor different physical quantities, the main ones being strain [4], pressure [5], acceleration [6], and mass [7]. These different measurements are needed in various fields of application, such as microfluidic [8], medicine [9], aerospace [10], and thin films deposition [11].

A review of main force sensors, at both the micro and macro scales, is reported by Bell [1]. Force sensors are mapped following their resolution and the maximum force they can

Manuscript received August 30, 2012; revised December 13, 2012; accepted January 17, 2013. Date of publication March 18, 2013; date of current version July 29, 2013. This work was supported by the Fonds de Recherche du Québec-Nature et Technologies International intership program. Subject Editor J. A. Yeh.

A. Poulin, R. St-Gelais, and Y.-A. Peter are with the Department of Engineering Physics, École Polytechnique de Montréal, Montréal, QC H3C 3A7, Canada (e-mail: alexandre-2.poulin@polymtl.ca; raphael.stgelais@polymtl.ca; yves-alain.peter@polymtl.ca).

A. L. Eichenberger is with the Federal Institute of Metrology METAS, CH3003 Bern-Wabern, Switzerland (e-mail: ali.eichenberger@metas.ch).

L. Thévenaz is with the Department of Electrical Engineering, École Polytechnique Fédérale de Lausanne, CH-1015 Lausanne, Switzerland (email: luc.thevenaz@epfl.ch).

Color versions of one or more of the figures in this paper are available online at http://ieeexplore.ieee.org.

Digital Object Identifier 10.1109/JMEMS.2013.2248252 measure. The ratio of these two parameters is a meaningful factor that can be used to characterize the performance of a sensor. Only few technologies allow high resolution measurements for loads ranging from 1 to $100 \mathrm{mN}$. Considering the ratio of the resolution to the maximum force as the figure of merit, it can be seen that macro sensors performance drops for loads below $100 \mathrm{mN}$. In contrast, most of the microsystem technologies cannot measure loads larger than $1 \mathrm{mN}$.

Piezoresistive and piezoelectric sensors appear to be the only candidates for high resolution measurements between 1 and $100 \mathrm{mN}$. However, the specific materials required for these technologies increase the complexity and the cost of fabrication [12]. Moreover, the output signal can be affected by electromagnetic interferences (EMIs) [10], and the electrostatic charge leakage in piezoelectric materials makes it impossible to realize static measurements [13]. These technologies are, therefore, not well suited for all applications. One example is the development of weighing devices that most of the time require static measurements. In addition, samples can exhibit magnetic properties or the device might be exposed to important electromagnetic noise as in aerospace applications.

This paper addresses the specific issue of high-precision weighing of samples in the range of $1-100 \mathrm{mN}$. An additional challenge related to this target is the large size of the samples compared to the microsystems. $100 \mathrm{mN}$ samples can easily be up to a few cubic centimeters. In this paper, we present a mass sensor based on an asymmetric Fabry-Perot (FP) resonator for high-precision weighing of macro samples. The in-plane cavity, composed of two silicon Bragg mirrors, is tuned when applying an external force on the device. Unlike piezoresistive and piezoelectric sensors, this approach is immune to EMI and allows static measurements. Only a few MEMS force sensors based on FP resonators have previously been published. A pressure sensor [4] and a shear stress sensor [8] were reported. They are respectively based on out-of-plane and in-plane resonators and do not involve distributed Bragg gratings (DBG). The use of DBG and an in-plane cavity in our design allows to achieve high reflectivity mirrors and makes design variations easier while keeping a simple fabrication process.

A third device, closer to the one we propose, was published recently [14]. The microsystem is based on a vertically etched silicon DBG and is presented as a force sensor. It was tested under forces up to $80 \mathrm{mN}$ and exhibits a maximum sensitivity of $14 \mathrm{~nm} / \mathrm{N}$. However, waveguides are used in the design and the tuning mechanism is completely different from ours. All 
optical components are attached together. It is the compression or extension of the silicon section between the two Bragg mirrors that modifies the optical path. Our device is a free space optical component. The gap between the two mirrors is thus an air layer and can be tuned over a much longer distance by allowing the in-plane motion of one of the mirrors.

None of these microsystems were used as weighing instruments. In order to facilitate the comparison, performances of our sensors will be presented as a function of the applied force rather than the applied mass. To our knowledge, we present the first microbalance based on a tunable FP resonator. It is also, to our knowledge, the first optical-MEMS developed for weighing of macro samples. The first section of this paper is divided in four subsections that, respectively, present the working principle of the device, the mechanical design, the optical design, and the fabrication process. The second section is composed of three subsections that, respectively, present the experimental setup, the optomechanical response of multiple devices, and a discussion on the sensor stability and resolution.

\section{DESIGN AND FABRICATION}

\section{A. Working Principle}

Fig. 1(a) shows a schematic of the proposed device. Four springs hold a suspended central section. The load patch (highlighted in red online) corresponds to the interaction surface. The force or weight to be measured must be applied on this area. A close-up view of the device is presented in Fig. 1(b) and shows the optical components in detail. Two Bragg mirrors of different reflectivity, separated by a gap of dimension $d_{\text {Gap }}$, form an asymmetric FP cavity. The parameters $d_{\text {Air }}$ and $d_{\mathrm{Si}}$, respectively, correspond to the thickness of silicon and air layers. The term asymmetric refers to the mirrors that are not of equal reflectivity. Facing the optical resonator, a fiber groove has been implemented to allow passive optical alignment. The optical fiber inserted in this groove, referred as the interrogating fiber, is used to monitor the reflection spectrum of the device.

The resonance wavelength $\lambda$ of an FP cavity can be tuned by modifying the optical length of the cavity. This can be achieved by changing the size of the gap or its refractive index. A change of $\lambda / 2$ leads to a spectral shift equal to the free spectral range (FSR) that corresponds to the spacing between each resonance peaks. Fig. 1 shows that one of the Bragg mirror is attached to the suspended section. This mirror is thus free to move along the optical axis of the cavity.

Consequently, the motion induced by an external force modifies the cavity length and the spectral position of the resonance. The force equilibrium condition leads to (1). As can be seen, the spectral shift $\Delta \lambda$ is linearly proportional to the applied force or mass

$$
F=m g=k \frac{\lambda \Delta \lambda}{2 F S R} .
$$

The in-plane design allows a large range of spring constants $k$, a parameter directly related to the sensitivity $S$ of the device. The sensitivity, defined as the ratio between the spectral shift and the applied force $(\mathrm{nm} / \mathrm{N})$, also depends on the FSR as

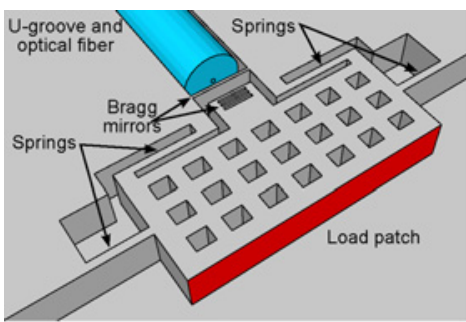

(a)

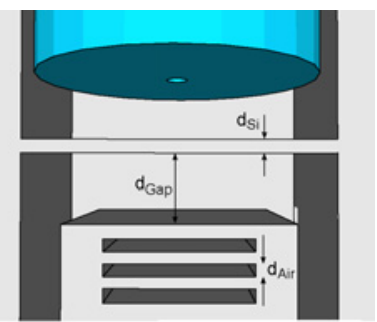

(b)
Fig. 1. (a) Schematic of the proposed device with (b) close-up view of the optical cavity. The parameters $d_{\mathrm{Air}}$ and $d_{\mathrm{Si}}$, respectively, correspond to the width of the air and silicon layers. The parameter $d_{\mathrm{Gap}}$ corresponds to the length of the FP cavity.

expressed by (2)

$$
S=\frac{\Delta \lambda}{m g}=\frac{2 F S R}{k \lambda} .
$$

\section{B. Optical Design}

Bragg mirrors correspond to a stack of alternating layers of high and low refractive indices. To obtain a reflection band centered at $\lambda$, the thickness of each layer $\left(d_{\mathrm{Air}}, d_{\mathrm{Si}}\right)$ must correspond to an odd integer of $\lambda / 4$. The bandwidth of the mirror is determined by the order of each layer and by the refractive index contrast. The orders of silicon and air layers are respectively defined as $m_{\mathrm{Air}}$ and $m_{\mathrm{Si}}$ in (3). Low orders and high refractive index contrast lead to larger bandwidth. Silicon is, therefore, a good candidate because it has a high refractive index contrast with air $(\Delta \mathrm{n}=2.45$ at $\lambda=1.55 \mu \mathrm{m})$. The lower limit on $m_{\mathrm{Air}}$ and $m_{\mathrm{Si}}$ is set by the lithography resolution, whereas the upper limit is set by the mirror bandwidth that must be at least as large as the targeted FSR

$$
\begin{aligned}
& m_{\text {Air }}=\frac{4 d_{\text {Air }}}{\lambda} n_{\text {Air }} \\
& m_{\mathrm{Si}}=\frac{4 d_{\mathrm{Si}}}{\lambda} n_{\mathrm{Si}} \\
& m_{\text {Gap }}=\frac{2 d_{\mathrm{Gap}}}{\lambda} n_{\text {Air }} .
\end{aligned}
$$

The reflectivity of Bragg mirrors is determined by the refractive index contrast and the number of periods (i.e., pairs of high and low refractive index layers.). To optimize the reflection signal we chose to work with an asymmetric cavity. The back-end mirror was designed to reach a maximal reflectivity. It was calculated that there is no significant improvement of the reflectivity above four periods (i.e., four silicon walls). This is due to the high refractive index contrast between layers and optical losses induced by surface roughness [15]. The simulations showed that the entrance mirror reflectivity is of great importance. The optical losses in the cavity are nonnegligible and the front mirror reflectivity must be optimized to get the highest signal, while maintaining a small enough full width at half maximum.

To obtain a resonance centered at $\lambda$, the length of an FP cavity $d_{\text {Gap }}$ must correspond to an integer of $\lambda / 2$. The gap order, defined as $m_{\text {Gap }}$ in (3), determines the free spectral range between each resonance. Low orders lead to larger 
TABLE I

DEVICES OPTICAL PARAMETERS

\begin{tabular}{ccccccc}
\hline $\begin{array}{c}m_{\text {Air }} \\
-\end{array}$ & $\begin{array}{c}d_{\text {Air }} \\
(\mu \mathrm{m})\end{array}$ & $\begin{array}{c}m_{\mathrm{Si}} \\
-\end{array}$ & $\begin{array}{c}d_{\mathrm{Si}} \\
(\mu \mathrm{m})\end{array}$ & $\begin{array}{c}m_{\mathrm{Gap}} \\
-\end{array}$ & $\begin{array}{c}d_{\mathrm{Gap}} \\
(\mu \mathrm{m})\end{array}$ & $\begin{array}{c}\text { FSR } \\
(\mathrm{nm})\end{array}$ \\
\hline 7 & 2.7 & 13 & 1.4 & 7 & 5.4 & 120 \\
\hline
\end{tabular}

FSR. For short cavities formed of Bragg mirrors, there is no simple equation that expresses the relation between these two parameters. It is due to the non-negligible penetration depth of light into the mirrors, which modifies the effective cavity length. In that case, the transfer-matrix method [16] must be used to determine the expected FSR. As mentioned previously, there is a linear relation between the sensitivity and the FSR. Therefore, the gap order must be minimized to increase the sensitivity. The lower limit on $m_{\text {Gap }}$ is set by the bandwidth of the mirrors that must cover the whole FSR.

Considering these different targets and limitations, the optical design parameters $\left(m_{\text {Air }}, m_{\mathrm{Si}}, m_{\text {Gap }}\right.$ and the number of periods) were optimized using a previously reported model [15]. This model takes into account not only the thickness and refractive indices of each layer but also the optical losses due to Gaussian beam divergence and surface roughness, thus allowing more accurate results. Roughness of $25 \mathrm{~nm}$ RMS was considered for all simulations. Table I shows the optimal Bragg dimensions and the resulting FSR. Two different designs were fabricated. The first uses the interface between the optical fiber and air as the entrance mirror. The second uses a Bragg mirror of 0.5 period (i.e., one silicon walls). For both designs, the back-end Bragg mirror is composed of four periods.

\section{Mechanical Design}

As this device will be used to weight macro samples, the dimensions of the load patch must be maximized. Our fabrication process restricts the thickness of the device layer and thus the height of the load patch. Its width can however take any value up to a few millimeters. The fabricated devices have a load area of $500 \mu \mathrm{m} \times 70 \mu \mathrm{m}$. This surface is still about three orders of magnitude smaller than the samples we need to weight. The solution to this constrain is detailed in Section III-A.

The design includes four springs to ensure its stability. Ideally, the only possible motion would be a translation along the $x$-axis that corresponds to the optical axis of the FP cavity. The mechanical configuration limits the amplitude of unwanted translation or rotation motions, while keeping one side of the device accessible to be used as the load area. The total spring constants of the structure in the $\mathrm{x}$ and $\mathrm{z}$ directions are given by (4) where the parameters $E, t, d$, and $l$, respectively, correspond to the Silicon Young modulus, the thickness, the width, and the length of the springs

$$
\begin{aligned}
& k_{x}=\frac{E t d^{3}}{l^{3}} \\
& k_{z}=\frac{E d t^{3}}{l^{3}} .
\end{aligned}
$$

Four different sets of springs were used for the fabricated devices. They are referred to as $S 1$ to $S 4$ and listed in Table II with the associated dimensions, minimum load, maximum load
TABLE II

DEVICES STRUCTURAL VARIATIONS

\begin{tabular}{cc|cccc}
\hline \multicolumn{2}{c|}{ Structural variations } & $S 1$ & $S 2$ & $S 3$ & $S 4$ \\
\hline$t$ & $(\mu \mathrm{m})$ & 70 & 70 & 70 & 70 \\
$d$ & $(\mu \mathrm{m})$ & 5.7 & 9.1 & 12.3 & 13.1 \\
$l$ & $(\mu \mathrm{m})$ & 145 & 145 & 100 & 100 \\
$k_{x}$ & $(\mathrm{kN} / \mathrm{m})$ & 2.5 & 12.7 & 25.3 & 126.5 \\
$k_{z}$ & $(\mathrm{kN} / \mathrm{m})$ & 384 & 656 & 1738 & 2972 \\
\hline Minimum load & $(\mathrm{mN})$ & 7.84 & 0 & 78.4 & 0 \\
Maximum load & $(\mathrm{mN})$ & 9.8 & 9.8 & 98 & 98 \\
Dynamic range & $(\mathrm{mN})$ & 1.96 & 9.8 & 19.6 & 98 \\
\hline
\end{tabular}

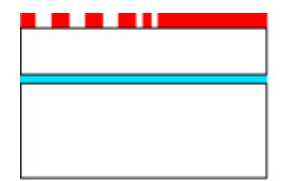

$\square: \mathrm{Si}$

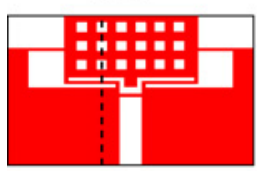

(a)
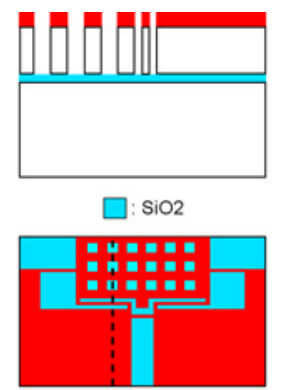

(b)

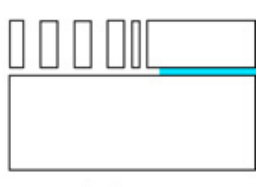

: Resist

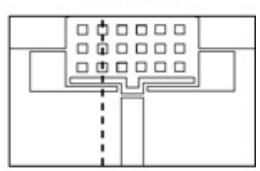

(c)
Fig. 2. Schematic of the device after the (a) photolithography, (b) DRIE, and (c) release steps. The upper and lower images respectively present side and top views of the device through its three fabrication steps. The side views are along the dotted lines visible in the lower images.

and dynamic range. The dynamic range corresponds to the mass needed to move the back-end mirror over a $\lambda / 2$ distance. As can be seen from (2), for a given FSR the dynamic range and sensitivity are inversely proportional. Therefore, a compromise needs to be reached between these two parameters. The springs $S 1$ and $S 2$ are designed to work under a load of $9.8 \mathrm{mN}(1 \mathrm{~g})$. The dynamic range of $S 1$ is however five times lower. On the other hand, its sensitivity is five times higher. The same relation exists between $S 3$ and $S 4$, which are designed to work under a maximum load of $98 \mathrm{mN}(10 \mathrm{~g})$. The out-of-plane spring constants were used to calculate the vertical motion of the suspended section induced by its own weight. Considering a mass of $12 \mu \mathrm{g}$, a vertical deflection of $0.3 \mathrm{pm}$ was calculated for the lowest $k_{z}$. There is therefore no risk for the suspended section to collapse or tilt and cause an important misalignment between the two mirrors. The high ratio of the in-plane spring constant to the out-of-plane spring constant (ranges from 25 to 150 depending on the device) minimizes the unwanted motion along the out-of-plane axis.

\section{Fabrication}

The out-of-plane design of the microsystem allows to minimize the number of fabrication steps and therefore increases the production rate of the process. The fabrication process we used is adjusted from [17] and involves only three steps, as can be seen in Fig. 2.

The substrate is a silicon-on-insulator $\langle 110\rangle$ wafer. The device layer and buried oxide are respectively 70 and $2 \mu \mathrm{m}$ thick. The device layer thickness ensures the interrogating fiber (Corning SMF28) core will be aligned within the top $15 \mu \mathrm{m}$ of the mirrors once inserted in the groove. The first step is a photolithography. Shipley SPR 7.0 resist is spun at $2500 \mathrm{rpm}$ 
TABLE III

DRIE PARAMETERS

\begin{tabular}{cc|cc}
\hline & & Passivation & Etching \\
\hline$t$ & $(\mathrm{~s})$ & 3 & 6 \\
$T$ & $\left({ }^{\circ} \mathrm{C}\right)$ & 20 & 20 \\
$\mathrm{O}_{2}$ & $(\mathrm{sccm})$ & 0 & 6.5 \\
$\mathrm{C}_{4} \mathrm{~F}_{8}$ & $(\mathrm{sccm})$ & 65 & 15 \\
$\mathrm{SF}_{6}$ & $(\mathrm{sccm})$ & 1 & 65 \\
$P_{\text {platen }}$ & $(\mathrm{W})$ & 10 & 25 \\
$P_{I C P}$ & $(\mathrm{~W})$ & 450 & 450 \\
Pressure & $($ mTorr $)$ & 15 & 15 \\
\hline \multicolumn{2}{c|}{ Number of cycles } & \multicolumn{3}{|c}{500} \\
\hline
\end{tabular}

for $60 \mathrm{~s}$ to obtain a $5.7 \mu \mathrm{m}$ thick layer. The second step is the deep reactive ion etching (DRIE) of the device. All optical and mechanical components are etched simultaneously. The DRIE parameters are shown in Table III. An oxygen plasma is used to strip the remaining photoresist. The final step is the release of the structure in $49 \% \mathrm{HF}$ during 15 min. Periodic holes are etched in the central section to facilitate the release step. The lateral oxide etch required is thus lowered by more than one order of magnitude. As a second utility, the periodic holes also lower the mass of the suspended section. Finally, critical point drying is used to avoid sticking of the structure. No significant distortion or anchoring effect was observed on any of the fabricated devices. The effect of possible internal stresses across the SOI substrate might have been limited by the thickness of the device layer.

Fig. 3 shows a scanning electron microscopy image of the fabricated device before the release step. The left inset shows a close-up view of the entrance mirror lower section. As can be seen, the last few microns were completely etched, which is partly due to a slight nonverticality of the etching profile. $0.5^{\circ}$ deviation was measured in $2.7 \mu \mathrm{m}$ wide trenches (equal to the Bragg mirrors air layer thickness $d_{\text {Air }}$ ). The etching profile is influenced by trench width and larger opening (i.e., the FP cavity and optical fiber groove) will most likely exhibit sidewalls with lower verticality. However, only the top $15 \mu \mathrm{m}$ are actually exposed to the incident light beam. In this region, the thickness of the silicon walls is close to the expected value. The right inset shows a close-up view of Bragg mirrors upper section. Trenches with sidewalls of optical quality surface were achieved by optimization of the DRIE process. $25 \mathrm{~nm}$ RMS surface roughness was measured by white light interferometry in $2.7 \mu \mathrm{m}$ wide trenches. Surface quality is influenced by trench width, and larger openings are expected to exhibit sidewalls with higher roughness. For this type of optical resonator, surface roughness is the main source of optical losses [15]. Nonverticality of the optical interfaces and divergence of light (which can be amplified by nonverticality in the Bragg grating) also increase optical losses.

\section{RESUltS AND DISCUSSION}

\section{A. Experimental Setup}

The fabricated sensors were characterized using the experimental setup shown in Fig. 4. The setup can be divided in three

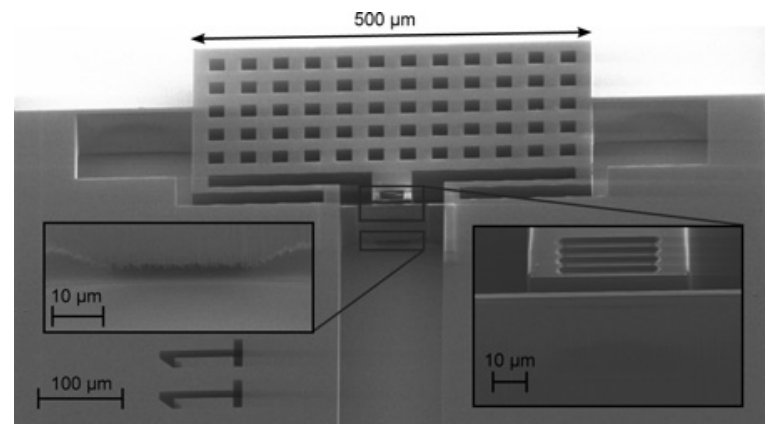

Fig. 3. Scanning electron microscopy image of a fabricated device. Right inset: close-up view of the asymmetric FP cavity. Left inset: the bottom of the first mirror has been etched due to a slight non-verticality of the process.

main sections. The first one allows to monitor the spectral response of the device. Light from an erbium broadband source, stabilized by a controller, is injected in an optical fiber and directed toward the microsystem for interrogation of the asymmetric FP cavity. The reflection spectrum of the cavity is coupled back into the fiber and an optical circulator, inserted between the broadband source and the device, redirects it toward the optical spectrum analyzer (OSA). The optical path presents many junctions and interfaces that can cause unwanted back reflections. An isolator has therefore been integrated at the exit of the broadband source.

The second section allows the alignment of the different setup components. The interrogating fiber is inserted in the fiber groove and aligned using a three-axis stage. We can see in Fig. 1(b) that a cavity is formed between the fiber and the first Bragg mirror when those two are not in contact. The end of the fiber was therefore cleaved at $8^{\circ}$ to minimize the parasitic effect of this cavity. It was done at the expense of increased optical losses. Ideally, an antireflective coating or an index matching gel would be used. Indeed, angle cleaved fibers were not used for devices where the interface between the optical fiber and air acted as the entrance mirror. The microsystem is placed on a two axis stage under a transparent plastic box that isolates the sensor from pressure variations in its surroundings. The samples are applied onto the sensor with the help of a component referred to as the force system. A three-axis stage is used to align the force system tip with the load patch of the device.

The third and final section allows loading of the microbalance. Our objective is to weigh macroscopic samples with a device exhibiting a microscopic sensing area. In order to make it possible we developed the force system, which significantly increases the sensing area of the device. The contact area of the force system tip is $0.1 \mathrm{~mm}^{2}$, whereas the area of the load pan is $176.7 \mathrm{~mm}^{2}$. The system is composed of an S-shaped structure with a rotation axis at the center of the vertical beam. As the horizontal beams are not of equal weight, the movable disks are used to equilibrate the system before samples are applied on the load pan. Due to the rotational motion, the resulting force exhibits an out-of-plane component. However, the vertical beam of the force system is over four orders of magnitude longer than the maximum in-plane displacement of the mirror. The maximum angle of rotation is therefore 


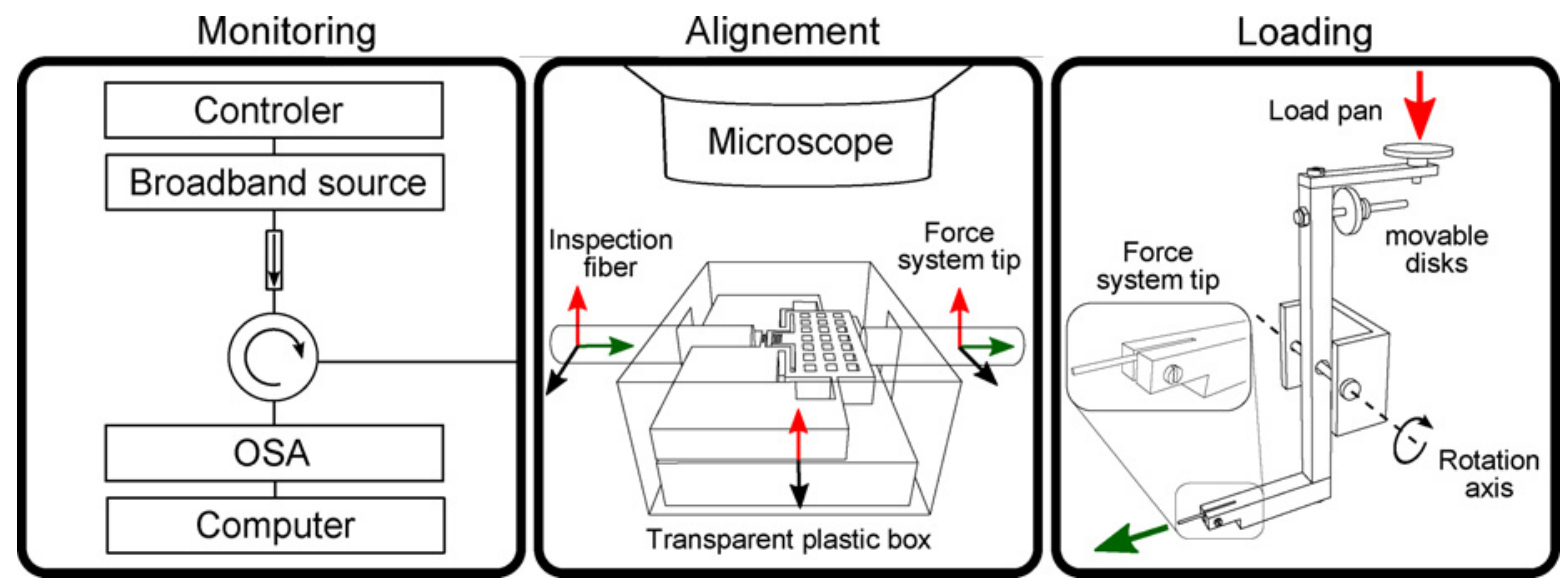

Fig. 4. Schematic of the experimental setup. It is composed of three main sections dedicated to the monitoring of the spectral response, the fine alignment of the different components, and the interaction between the macroscopic samples and the microscopic sensor.

in the order of one thousandth of a degree. Consequently, the out-of-plane component of the resulting force is small enough to be neglected. Larger errors can be induced by a misalignment of the Force system. Its tip should be aligned to the center of the Load patch, perpendicularly to the contact surface. Offsets from the center or an angular misalignment induce a tilt between the two Bragg mirrors that can result in increased optical losses and spectral shift of the FP resonance. The alignment was done by visual inspection and lead to an approximated resolutions of $20 \mu \mathrm{m}$ for the centering and $10^{\circ}$ for the angular positioning. A second approach based on a motion translation system could be used for further developments [18]. It would allow enlarging significantly the load area of the microsystem, thus eliminating the need for a force system and allowing a more integrated sensor.

\section{B. Optomechanical Characterization}

Two optical designs and four structural variations were presented in Sections II-B and II-C, respectively. Based on these variations, devices of different configurations were fabricated and characterized. The sensitivity of the resulting sensors corresponds to the ratio of the FSR to the dynamic range. It is therefore determined by the initial length of the cavity and the spring constant of the mechanical structure. For the first optical design, the cavity is formed by two Bragg mirrors. Its initial length is therefore fixed, just as its spring constant. Consequently, the expected sensitivity can be calculated and compared with the experimental results. For the second optical design, the cavity is formed by the fiber/air interface and a high reflectivity Bragg mirror. In that case, the cavity length is determined during the alignment and is hard to evaluate visually. It is therefore not possible to provide the expected sensitivity of devices based on this design.

For each device, the spectral response was monitored under increasing loads. The spectral shift of the resonance was then reported in function of the applied load. A linear relation between these parameters is expected. Its slope corresponds to the sensor sensitivity. Fig. 5 presents the typical response of a device. It shows a superposition of multiple spectra measured under different loads. As expected, a resonance shift

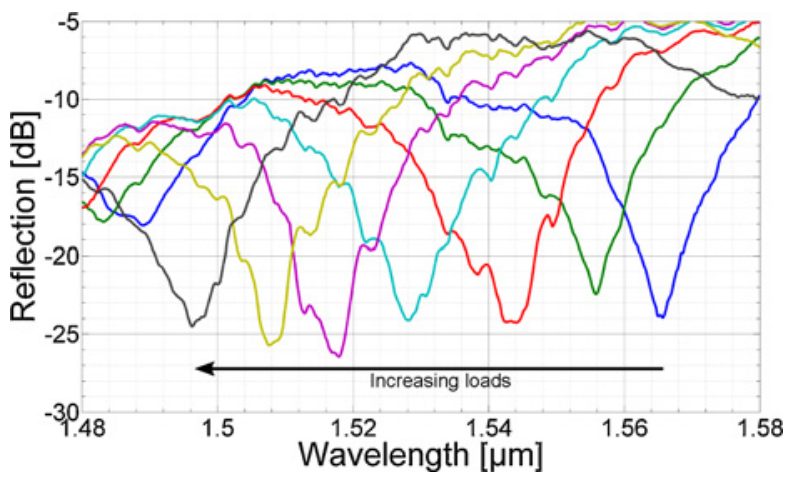

Fig. 5. Superposition of multiple spectra showing the sensor response under increasing loads. The device is based on the structural variation $S 2$ and its entrance mirror is a Bragg reflector.

toward shorter wavelengths is observed as the applied load increases. This shift corresponds to a decrease of the cavity length. For this particular device, the cavity is formed of two Bragg mirrors. The geometry of the springs corresponds to the second structural variation $S 2$ listed in Table II, from which we expect a sensitivity of $12.24 \mathrm{~nm} / \mathrm{mN}$. It can be seen that optical losses and resonance spectral width are increasing with the applied load. It is most likely due to an increasing tilt between the two Bragg mirrors, induced by a misalignment of the Force system tip. For the maximum load, the resonance still exhibits an isolation of $15 \mathrm{~dB}$. The operation of the sensor is therefore not compromised. Fig. 6 reports the spectral shift of the device in function of the applied load. An experimental sensitivity of $11.56 \mathrm{~nm} / \mathrm{mN}$ is obtained for the sensor, which is in good agreement with the expected value. The misalignment of the Force system does not seem to significantly affect the sensor response that appears to be in good correlation with the expected linear behavior.

Using the same methodology, devices based on four different configurations were characterized. Details of the configurations and experimental results are reported in Table IV. As can be seen from this table, structural variations $S 1$, $S 2$, and $S 3$ were implemented with an optical resonator composed of two Bragg mirrors. The cavity length is therefore 


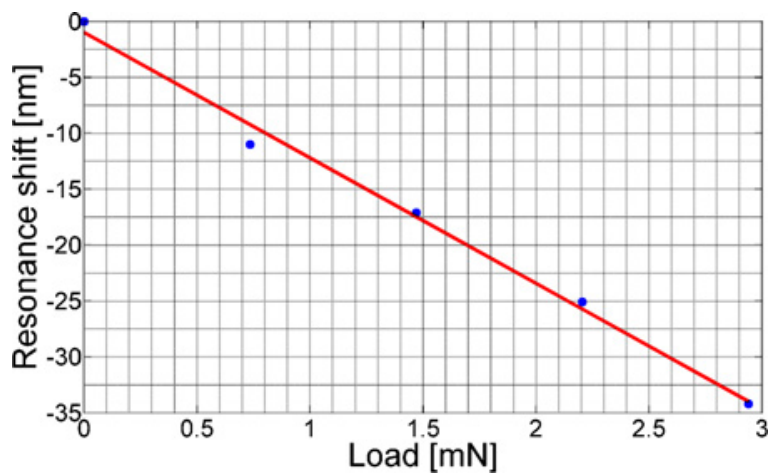

Fig. 6. Spectral shift of the resonance under increasing loads. The slope of the linear regression gives a sensitivity of $11.56 \mathrm{~nm} / \mathrm{mN}$. The device is based on the structural variation $S 2$ and its entrance mirror is a Bragg reflector.

TABLE IV

DEVICES CONFIGURATIONS AND EXPERIMENTAL RESUlTS

\begin{tabular}{lc|cccc}
\hline \multicolumn{2}{c|}{ Devices configurations } & $D 1$ & $D 2$ & $D 3$ & $D 4$ \\
\hline \multicolumn{2}{c}{ Structural variations } & $S 1$ & $S 2$ & $S 3$ & $S 4$ \\
\multicolumn{2}{c}{ Low reflectivity mirror } & Bragg & Bragg & Bragg & Fiber/air \\
\hline Theo. sensitivity & $(\mathrm{nm} / \mathrm{mN})$ & 61.22 & 12.24 & 6.12 & - \\
Exp. sensitivity & $(\mathrm{nm} / \mathrm{mN})$ & 67.69 & 11.56 & 5.92 & 0.51 \\
Dynamic range & $(\mathrm{mN})$ & 1.48 & 6.57 & 14.7 & 62 \\
Abs. resolution & $(\mu \mathrm{N})$ & 0.15 & 0.86 & 1.69 & 19.61 \\
Rel. resolution & $(\mathrm{ppm})$ & 101 & 131 & 115 & 316 \\
\hline
\end{tabular}

fixed and the experimental results can be compared with the expected sensitivities. Only one of the reported devices uses the fiber/air interface as the entrance mirror. In this configuration, it was hard to achieve small cavity lengths due to the limited precision of the alignment setup. Therefore, this optical design was more appropriate for a device of lower sensitivity. Consequently, it was implemented with the least sensitive structural variation $S 4$ to maximize the spectrum covered by these four devices. Devices $D 1$ and $D 2$ were tested under masses up to $9.8 \mathrm{mN}(1 \mathrm{~g})$, whereas devices $D 3$ and $D 4$ were tested under masses up to $98 \mathrm{mN}(10 \mathrm{~g})$. The sensors' experimental sensitivities cover more than two orders of magnitude, ranging from 0.51 to $67.69 \mathrm{~nm} / \mathrm{mN}$.

As can be seen from Table IV, there is a good correlation between theoretical and experimental sensitivities. The small deviations we observe come from mismatches between experimental and nominal dimensions of the devices. Diffraction of light during photolithography and underetch during bulk micromachining cause these mismatches and tend to increase the width of the trenches. Consequently, these sources of errors increase the cavity length and lower the width of the springs. It is interesting to note that the resulting alterations of optical and mechanical structures have opposite effects on the device sensitivity. An increase of the cavity length (higher FSR) leads to a lower sensitivity, whereas a decrease of the springs width (lower spring constant $k$ ) leads to a higher sensitivity. As can be seen from Table IV, experimental sensitivity of device $D 1$ is lower than expected, whereas experimental sensitivities of devices $D 2$ and $D 3$ are higher than expected. These devices'optical configurations are identical. Therefore, the relative effect of the cavity length mismatch on the device

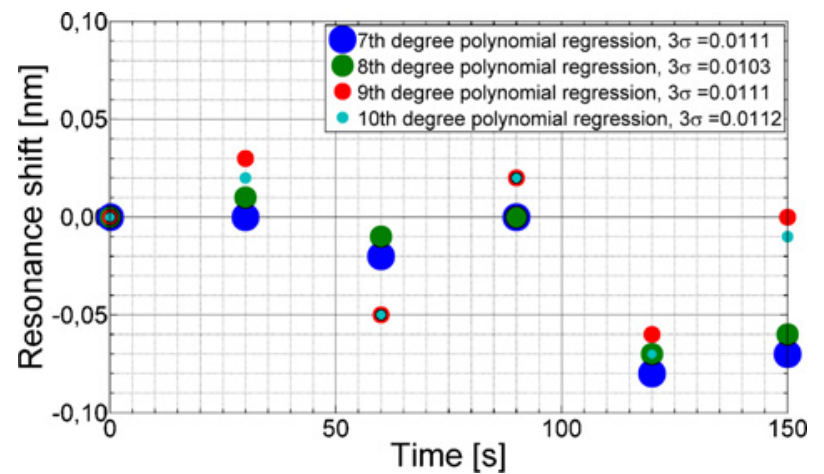

Fig. 7. Evolution of the resonance central position (extracted from polynomial regressions of different degrees) over time. It appears, from the standard deviation of the results, that the position of the resonance can be determined with a precision of $0.01 \mathrm{~nm}$.

sensitivity is constant from one device to another. On the other hand, the width of the springs increases from devices $D 1$ to $D 3$. Therefore, the relative effect of the springs width mismatch on the device sensitivity varies from one device to another. The mechanical effect is dominant for device $D 1$, whereas the optical effect is dominant for devices $D 2$ and $D 3$.

\section{Resolution and Stability}

In order to evaluate the sensor resolution, we measured the stability of the FP resonance using the following methodology. Firstly, the reflection spectrum of the resonator was monitored and recorded every $30 \mathrm{~s}$ over a time interval of $2.5 \mathrm{~min}$. Second, each of these spectra were run through a MATLAB algorithm to locate and extract the resonance. Polynomial functions of various degrees were then used to fit the resonance shape and extract its central position. Finally, the standard deviation was calculated for each degree of polynomial regression. The precision of the spectral measurement was defined as three times the lowest standard deviation. Fig. 7 reports the experimental results. The lowest standard deviation was obtained with a 7th degree polynomial regression and gives a precision of $0.01 \mathrm{~nm}$, which is smaller than the OSA resolution. The measurement is therefore most likely limited by the equipment and not by the device in itself. The absolute resolution of the sensor is obtained by multiplying its sensitivity by the precision of the spectral measurement. The experimental results are presented in Table IV and cover more than two orders of magnitude, ranging from 19.61 to $0.15 \mu \mathrm{N}$.

An additional meaningful parameter is presented. It corresponds to the ratio of the absolute resolution to the dynamic range. It is also equivalent to the ratio of the spectral resolution to the FSR. Expressed in part per million (ppm), it gives the maximum relative resolution of the sensor. The lower performance of device $D 4$ can be explained by its optical design. Its longer cavity leads to a shorter FSR and therefore to lower relative resolution. The smaller variations between the three other devices come from the fabrication process. As explained previously, small deviations from the devices' nominal dimensions are observed. These deviations are not constant from one device to another. The maximum relative resolution of $100 \mathrm{ppm}$ was obtained with device $D 1$. As we are 
limited by the OSA resolution, we can claim that the maximum relative resolution of the sensor is below $100 \mathrm{ppm}$.

Thermal effects were not significant enough to be observed during the experimental characterization where no effort was made to keep the temperature constant. Moreover, the optical and mechanical variations induced by temperature changes are much slower than the time required for a single measurement. Therefore, a calibration could be done in real time using a reference device for which the only unknown is the temperature (i.e., zero or constant load). Thermal effects are expected to become more important as the sensitivity of the device increase. Consequently, an in-depth study of the temperature dependence might be required for the device's next generations.

\section{CONCLUSION}

In this paper, we proposed MEMS-based technology for high-precision weighing of macro samples in the range of 0.1 to $100 \mathrm{mN}$. We presented what is, to our knowledge, the first optical microbalance based on a tunable FP cavity for weighing of macro samples. The device was structured on a silicon-on-insulator substrate. Its in-plane design allowed a simple and low cost fabrication process. The working principle, based on the mechanical deformation of an optical resonator, was immune to electromagnetic noise and allowed static measurement. Simulations were performed to predict the optical and mechanical behaviors of the device. Devices based on four different configurations were successfully fabricated and characterized.

We demonstrated that our optical microsystem can be used for high-precision weighing of macro samples. Masses up to $98 \mathrm{mN}$ (10 g) were successfully applied on the sensor and weighed. Devices with experimental sensitivities ranging from 0.51 to $67.69 \mathrm{~nm} / \mathrm{mN}$ and absolute resolutions ranging from 0.15 to $19.61 \mu \mathrm{N}$ were presented. The covered range could be further increased by simple modifications of the spring dimensions. The maximum relative resolution of the sensor is below $100 \mathrm{ppm}$ and is limited by the OSA resolution. Alternative methods such as laser locking could be used to track the resonance. It would most likely improve the sensor resolution, but at the expense of lower dynamic range.

The maximum sensitivity of our sensor was more than three orders of magnitude higher than previously reported vertically etched silicon DBG force sensor [14]. For similar maximum load and dynamic range, our device (device D4) sensitivity appeared to be four times higher. Moreover, device $D 4$ used the fiber/air interface as the entrance mirror, which was the least sensitive optical design. Its sensitivity could therefore be easily increased by a factor three.

\section{REFERENCES}

[1] D. Bell, T. Lu, N. Fleck, and S. Spearing, "MEMS actuators and sensors: Observations on their performance and selection for purpose," J. Micromech. Microeng., vol. 15, no. 7, p. S153, 2005.

[2] J. Shieh, J. Huber, N. Fleck, and M. Ashby, "The selection of sensors," Progress Mater. Sci., vol. 46, nos. 3-4, pp. 461-504, 2001.
[3] W. Eaton and J. Smith, "Micromachined pressure sensors: Review and recent developments," Smart Mater. Struct., vol. 6, no. 5, p. 530, 1997.

[4] Y. Gianchandani and K. Najafi, "Bent-beam strain sensors," J. Microelectromech. Syst., vol. 5, no. 1, pp. 52-58, 1996.

[5] Y. Kim and D. Neikirk, "Micromachined Fabry-Perot cavity pressure transducer," IEEE Photonics Technol. Lett., vol. 7, no. 12, pp. 14711473, Dec. 1995

[6] J. Marty, A. Malki, C. Renouf, P. Lecoy, and F. Baillieu, "Fibreoptic accelerometer using silicon micromachining techniques," Sens. Actuators A, Phys., vol. 47, no. 1, pp. 470-473, 1995.

[7] M. Muratsugu, F. Ohta, Y. Miya, T. Hosokawa, S. Kurosawa, N. Kamo, and H. Ikeda, "Quartz crystal microbalance for the detection of microgram quantities of human serum albumin: Relationship between the frequency change and the mass of protein adsorbed," Anal. Chem., vol. 65 , no. 20, pp. 2933-2937, 1993.

[8] F. Tseng and C. Lin, "Polymer MEMS-based Fabry-Perot shear stress sensor," IEEE Sensors J., vol. 3, no. 6, pp. 812-817, Dec. 2003.

[9] R. Wolthuis, G. Mitchell, E. Saaski, J. Hartl, and M. Afromowitz, "Development of medical pressure and temperature sensors employing optical spectrum modulation," IEEE Trans. Biomed. Eng., vol. 38, no. 10, pp. 974-981, Oct. 1991.

[10] H. Shea, "Reliability of MEMS for space applications," in Proc. SPIE, vol. 6111. 2006, p. 61110A.

[11] S. Martin, V. Granstaff, and G. Frye, "Characterization of a quartz crystal microbalance with simultaneous mass and liquid loading," Anal. Chem., vol. 63, no. 20, pp. 2272-2281, 1991.

[12] D. DeVoe, "Piezoelectric thin film micromechanical beam resonators," Sens. Actuators A, Phys., vol. 88, no. 3, pp. 263-272, 2001.

[13] O. Jeong and S. Yang, "Fabrication of a thermopneumatic microactuator with a corrugated p+ silicon diaphragm," Sens. Actuators A, Phys., vol. 80 , no. 1 , pp. $62-67,2000$.

[14] K. Reck, E. Thomsen, and O. Hansen, "MEMS Bragg grating force sensor," Opt. Express, vol. 19, no. 20, pp. 19 190-19198, 2011.

[15] R. St-Gelais, A. Poulin, and Y. Peter, "Advances in modeling, design, and fabrication of deep-etched multilayer resonators," J. Lightwave Technol., vol. 30, no. 12, pp. 1900-1908, Jun. 2012.

[16] H. Macleod, Thin-Film Optical Filters. New York, NY, USA: Taylor \& Francis, 2001.

[17] J. Masson, R. St-Gelais, A. Poulin, and Y. Peter, "Tunable fiber laser using a MEMS-based in plane Fabry-Pérot filter," IEEE J. Quantum Electronics, vol. 46, no. 9, pp. 1313-1319, Sep. 2010.

[18] A. Poulin, "Coupled electro-mechanical transducers for vertical to horizontal motion translation," in Proc. Symp. Des., Test, Integration Packaging MEMS/MOEMS, Apr. 2012, pp. 240-243.

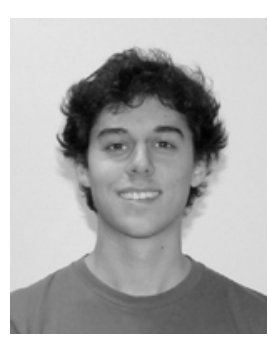

Alexandre Poulin received the M.Sc. degree in engineering physics from the École Polytechnique de Montréal, Montreal, QC, Canada, in 2012. During his Master's studies, he completed a research internship at the École Polytechnique Fédérale de Lausanne, Lausanne, Switzerland, and the Federal Institute of Metrology METAS, Switzerland, with the support of a FQRNT international internship award. He is currently pursuing the Ph.D. degree in microsystems and microelectronics at the École Polytechnique Fédérale de Lausanne.

His current research interests include microelectromechanical systems, microphotonics, and electroactive polymers for development of sensors and actuators.

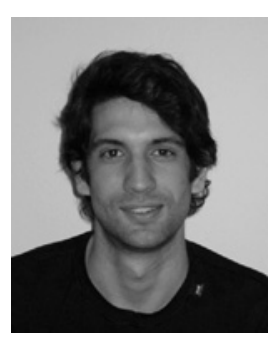

Raphael St-Gelais received the B.Eng. and Ph.D. degrees in engineering physics from the École Polytechnique de Montreal, Montreal, QC, Canada, in 2007 and 2012, respectively .

He was an Engineering Intern with Dalsa Semiconductor (now Teledyne Dalsa), Bromont, QC, Canada, before joining the Microphotonics Laboratory, École Polytechnique de Montreal, in 2008. Since 2013, he has been a Post-Doctoral Associate with the Cornell Nanophotonics Group, Cornell University, Ithaca, NY, USA. His current research interests include optical sensors, optical microelectromechanical systems, integrated optics, nanophotonics, and optofluidics. 


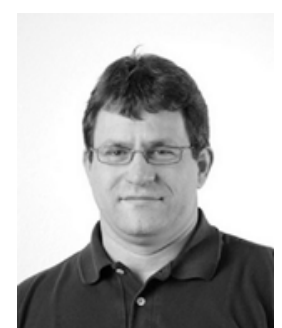

Ali L. Eichenberger received the Ph.D. degree in experimental condensed matter physics from the University of Neuchâtel, Neuchâtel, Switzerland, in 1997, studying disordered Josephson junction arrays.

From 1997 to 2000, he was a Guest Scientist at the National Institute of Standards and Technology, Boulder, CO, USA, in the Nanoscale Cryoelectronics Group, working on the development of a capacitance standard based on single electron tunneling devices. He joined the Federal Institute of Metrology METAS, Bern, Switzerland, in 2000 to work on the watt balance project.

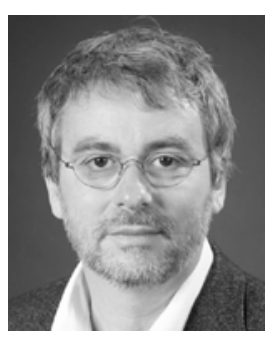

Luc Thévenaz (SM'12) received the M.Sc. and $\mathrm{Ph} . \mathrm{D}$. degrees in physics from the University of Geneva, Geneva, Switzerland, in 1982 and 1988, respectively.

In 1988, he joined the Swiss Federal Institute of Technology of Lausanne (EPFL), Lausanne, Switzerland, where he currently leads a research group involved in photonics, namely, fiber optics and optical sensing. His research topics include Brillouin-scattering fiber sensors, slow and fast light, nonlinear fiber optics, and laser spectroscopy in gases. He achieved with his collaborators the first experimental demonstration of optically controlled slow and fast light in optical fibers, and is at the origin of innovative configurations for Brillouin distributed fiber sensors, such as generation of the signal wave using modulation sidebands and Brillouin dynamic gratings. During his career, he has worked with Stanford University, Stanford, CA, USA, the Korea Advanced Institute of Science and Technology (KAIST), Tel Aviv University, Tel Aviv, Israel, and the University of Sydney, Sydney, Australia. In 2000, he co-founded the company Omnisens, which develops and commercializes advanced photonic instrumentation.

Dr. Thévenaz is a Fellow of the Optical Society of America.

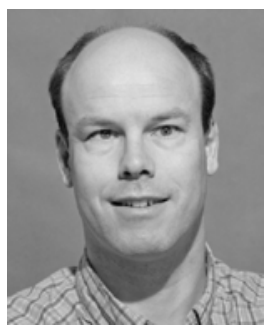

Yves-Alain Peter (S'93-M'03-SM'07) received the M.Sc. degree in physics and the Dr.Sc. degree in sciences from the University of Neuchâtel, Neuchâtel, Switzerland, in 1994 and 2001, respectively.

In 1995, he was a Research Associate with the Department of Medical Radiobiology, Paul Scherrer Institute, Switzerland. From 1995 to 2001, he was a Graduate Research Assistant with the Applied Optics Group, Institute of Microtechnology, University of Neuchâtel. From 2001 to 2003, he was a Post-Doctoral Researcher with the Microphotonics Group, Stanford University, Stanford, CA, USA. From 2003 to 2004, he was a Research and Development Engineer and a Project Leader with the Swiss Center for Electronics and Microtechnology, Switzerland. He is currently an Associate Professor with the Department of Engineering Physics, École Polytechnique de Montréal, Montréal, QC, Canada. His current research interests include microphotonics and microoptoelectromechanical systems. 